\title{
Improved spot morphology for printed glycan arrays
}

\author{
Maksim Navakouski ${ }^{1}$, Nadezhda Shilova', Nailya Khasbiullina ${ }^{1}$, Alexey Feofanov¹,2, Elena Pudova ${ }^{3}$, \\ Kowa Chen ${ }^{4}$, Ola Blixt ${ }^{4}$, Nicolai Bovin ${ }^{1}$ \\ 'Shemyakin-Ovchinnikov Institute of Bioorganic Chemistry, Russian Academy of Sciences, Miklukho- \\ Maklaya 16/10, 117997 Moscow, Russia, ²Biology Faculty, Lomonosov Moscow State University, Moscow, \\ 119992, Russia, ${ }^{3}$ Central Research Institute of Epidemiology, Federal Service for Supervision of Consumer \\ rights Protection and Human Well-Being, Novogireevskaia 3a, 111123 Moscow, Russia, ${ }^{4}$ Department of \\ chemistry, University of Copenhagen, t422, Thorvaldsensvej 40, 1870, Frederiksberg, Denmark.
}

\begin{abstract}
BioTechniques 64:110-111 (March 2018) doi 10.4155/btn-2017-0111
Keywords: printed glycan array; spots morphology; glycans; microarray; lab-on-chip
\end{abstract}

Despite considerable success studying glycan-binding proteins using printed glycan arrays (PGAs), unambiguous quantitation of spot intensities by fluorescent readers remains a challenge. The main obstacles are the varying spot shape and size and in-spot fluorescence distribution caused by uneven drying of the printed drops. Two methods have been suggested for solving this problem: using polymeric glycoconjugates, which makes it possible to equalize the physicochemical properties (hydrophobicity, charge, and size) of different glycans, and applying a glycan solution on a slide coated with a thin oil mask, which hinders evaporation of the drop. Both approaches yield spots with similar sizes and an even distribution of the signal across the spot and are likely to be useful for improving the prints of other classes of molecules.

The design of the most common printed glycan array (PGA) used today [3] meets many requirements for a quantitative assay, such as a wide dynamic range, low background, and a sufficient number of repeats for each spot. On the other hand, practical use of this and similar PGAs has revealed a number of factors that hinder quantitative estimation and comparison of data obtained using different printers, printing conditions, and reading or calculation methods. We considered one of these factors, heterogeneities of the size and shape of the spots, and the resulting signal distribution across the spot (Figure 1) in this study.

In contrast to the measurement of fluorescence in a solution, interpreting surface scanning results from a fluorescent reader is not strictly objective; it depends on the researcher's choice of calculation algorithm. The general intensity of spots can be estimated using 'fixed circles' and 'adaptive circles' approaches. In the former, a ring of a fixed diameter (the number of pixels inside each ring is the same), is measured for calculating of all of the spots. With adaptive circles, the software adjusts the ring to the spot border according to its algorithms such that the number of pixels inside the circle is different for each spot depending on its size. Figure 2 shows the comparison of data from the same array calculated by these two methods. Dramatic differences were observed for some glycans, especially for \#527 (3-O-Su-LeC), which was one of the most problematic glycans, forming spots with a vast halo.

Since there is no justified criterion for regarding any of these methods as correct, printing methods need to ensure reliable spots. We tested several approaches for solving this problem and found two of them that yielded the desired results.

\section{Materials and methods}

\section{Reagents}

Amino-spaced glycans and polyacrylamide glycoconjugates were from Lectinity Holdings (Russia). Other materials used were mineral oil (Tatchimfarmpreparaty, Russia), fructose, polyvinylpyrrolidone (400 kDa), glycerol triacetate (triacetine), diisopropylethyl amine (Fluka, Switzerland); boric acid (LUMI, Russia); 3-[(3-cholamidopropyl)dimethylammonio]-1-propanesulfonate (CHAPS), hexadecanol, sucrose, poly(ethylene oxide) (400 kDa), inositol, triethylamine, 1-butyl-3-methy-

\section{METHOD SUMMARY}

Two approaches improve spot morphology: 1) printing of polyacrylamide-linked glycans instead of monomeric glycans; 2) printing glycans in any form on a slide coated with a thin oil mask, which prevents evaporation of the drop. 


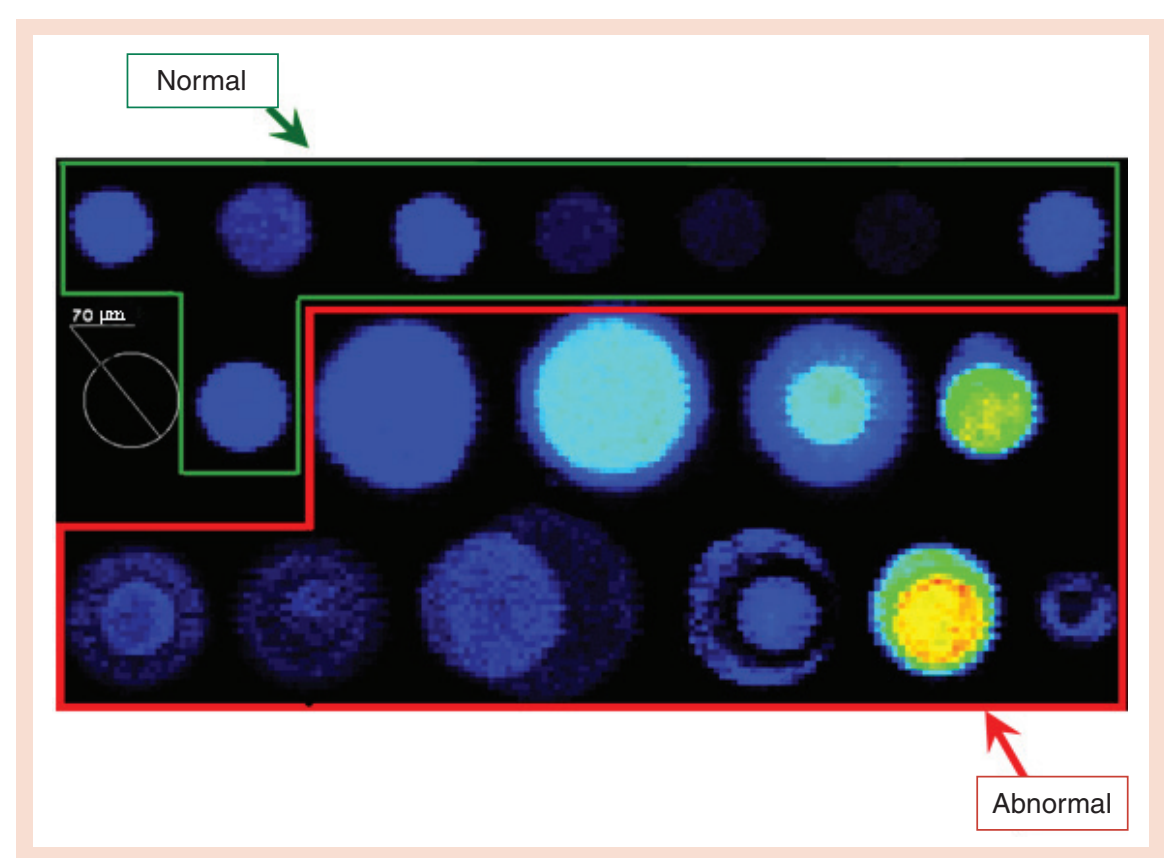

Figure 1. Heterogeneity of PGA spots developed with antibodies. Spots were taken from the same print, which was performed on NHS-activated slide H (Schott Nexterion, Germany). Spots with similar sizes and a homogenous signal distribution are considered normal. Large or small spots containing a halo, donut, etc. are considered abnormal.

limidazolium tetrafluoroborate, betaine, dextran (40 kDa), NP40, dodecan, ethanolamine, Tween-20 (SigmaAldrich, USA); TritonX100, agarose, bovine serum albumin (Serva, USA); $\mathrm{NaCl}$ (Dia-M, Russia); biotinylated secondary goat antibodies against human IgG + $\lg \mathrm{M}+\operatorname{Ig} \mathrm{A}$ (Thermo Sci, USA); streptavidin fluorescently labeled with Alexa 555 (Invitrogen, USA); complex immunoglobulin preparation (CIP) containing $50 \%$ of $\lg G, 25 \%$ of $\lg M$, and $25 \%$ of $\lg A$
(Immuno-gem, Moscow, Russia); and NHS-activated glass slides H (Schott Nexterion AG, Germany).

\section{Equipment}

A ScanArray Gx (PerkinEImer, Inc. USA) fluorescent microarray scanner was used for fluorescence measurements. An IX81 (Olympus, Japan) confocal microscope was used for 3D scanning of the spots at a magnitude of 500x and laser excitation at a wavelength of $543 \mathrm{~nm}$. A Nano-

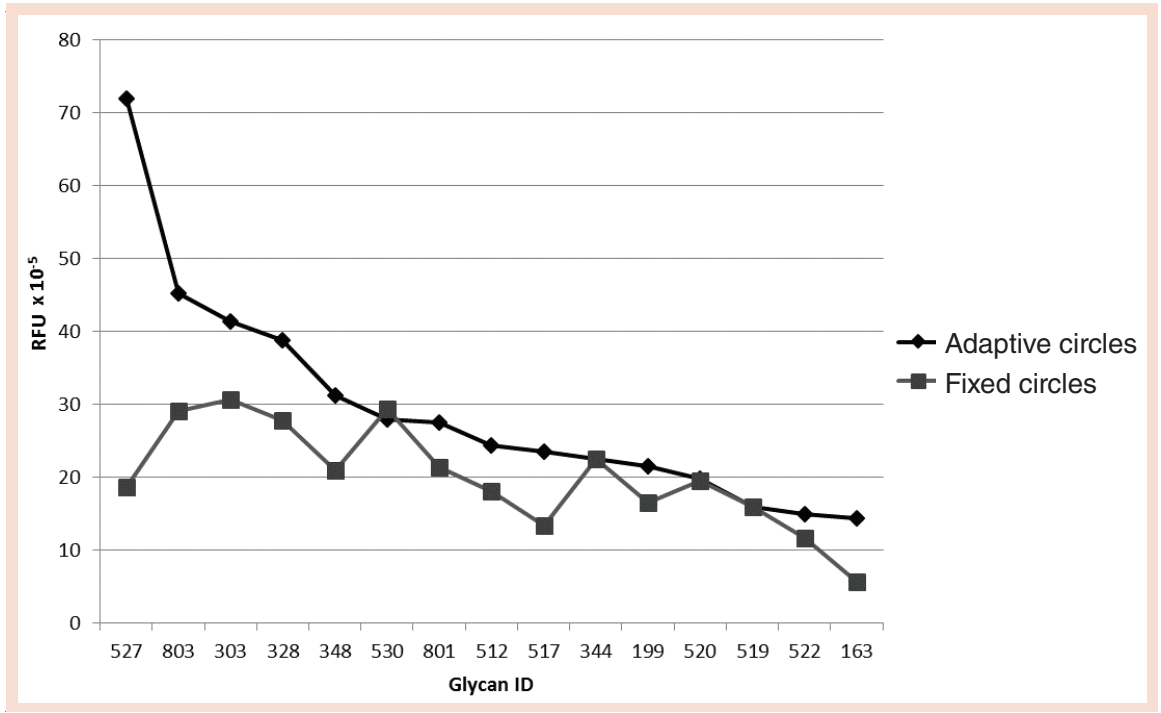

Figure 2. Comparison of two quantitation methods for the same glycan array. The intensities for the top 15 glycans calculated as a multiplication of the mean signal by the number of pixels per spot. The largest difference was observed for glycan \#527 (3-O-Su-Lec).
Plotter 2.1 (GeSim, Germany) piezoarrayer equipped with a Nano-Tip J was used for non-contact printing. A Biorobotics MicroGrid II (Digilab, USA) microarrayer equipped with SMP3B pins was used for contact printing.

\section{Printing procedures}

$50 \mu \mathrm{M}$ solutions of amino-spaced glycans in a DMSO-triacetine mixture (1:1) containing $1 \%$ of diisopropylethyl amine were printed onto NHS-activated slides $\mathrm{H}$ with the use of a non-contact printer at room temperature and ambient humidity of about $30 \%$. The slides were preliminarily coated with $300 \mu \mathrm{l}$ of mineral oil mixed with dodecan (2:1, $\mathrm{v} / \mathrm{v})$. A drop of solution pressed out of the capillary, breaks through the layer of the mask and deposits on the slide surface. Quality control was performed by means of a camera system (preand post-drop control). The chips were incubated at room temperature for $1 \mathrm{~h}$ after printing. Then the oil was washed away with phosphate-buffered saline (PBS) containing 1\% of Tween 20 and the chips were blocked with an ethanolamine (25 mM)-boric acid (100 $\mathrm{mM}$ ) buffer, $\mathrm{pH} 8.5$, containing $0.2 \%$ Tween-20 for $1 \mathrm{~h}$ at room temperature while gently rocking. The estimated mask thickness was about $16 \mu \mathrm{m}$.

Solutions of 30-kDa PAA glycoconjugates containing 20 mol\% of glycan (glycan concentration, $50 \mu \mathrm{M}$ ) in 300 mM sodium phosphate buffer ( $\mathrm{pH}$ 8.5) supplemented with $18 \%$ fructose and $0.005 \%$ Tween-20 were printed at room temperature and a humidity of $50 \%$ by means of the contact microarrayer onto $\mathrm{NHS}$-activated slides $\mathrm{H}$. The workability of the pins was tested before printing using the so-called pre-slide; all of the printed slides were visually inspected for missing spots. After printing, the chips were incubated at room temperature for $1 \mathrm{~h}$ at a humidity of $70 \%$. Then the activated groups were blocked with ethanolamine (25 mM)-boric acid (100 $\mathrm{mM}$ ) buffer, $\mathrm{pH} 8.5$, containing $0.2 \%$ Tween-20 for $1 \mathrm{~h}$ at room temperature while gently rocking.

\section{Assay of anti-glycan antibodies} Chips with the printed set of monomeric glycans or the corresponding PAA conjugates were incubated in the presence of CIP (1 mg/ml in PBS), $1 \%$ 
bovine serum albumin (BSA), and $1 \%$ Tween-20 for $1.5 \mathrm{~h}$ at $37^{\circ} \mathrm{C}$ while gently rocking; then the chips were washed with PBS containing $0.05 \%$ Tween-20, and biotinylated secondary goat antibodies against human $\lg G+\lg M+$ IgA (1:100) were added. After that, the chips were incubated for $1 \mathrm{~h}$ at $37^{\circ} \mathrm{C}$ while gently rocking and then they were washed as described above. A solution of streptavidin fluorescently labeled with Alexa 555 and diluted to 1:1000 in PBS containing $0.1 \%$ Tween-20 was added, and the mixture was incubated for $0.5 \mathrm{~h}$ at room temperature in the dark. Then the chips were washed as described above, washed with water, and scanned at a resolution of $5 \mu \mathrm{m}$ or examined under a standard trinocular microscope (Zeiss). The images were studied using the ProScanArray v.4.0 software (PerkinElmer, USA). 3D imaging of the microscopic data was performed using Huygens ${ }^{\circledR}$ Essential software (SVI, The Netherlands).

\section{Results and discussion}

Figure 1 shows highly magnified images of typical spots with (1) obvious size deviations, (2) a vast halo around the main central core, or (3) an intense core inside the spot with a uniform signal intensity over the entire area. The results were obtained for contact printing of glycans as aminoalkyl glycosides. Development with biotinylated secondary antibodies and fluorescently labeled streptavidin was carried out after

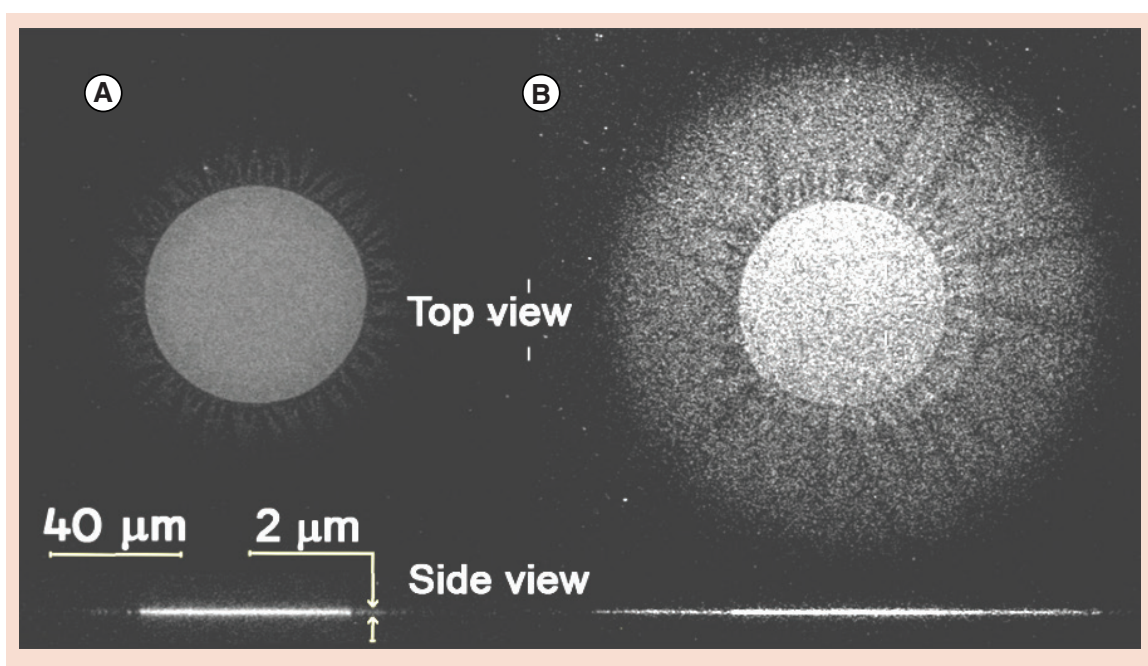

Figure 3. The top and side views of typical spot images at a high resolution. Images from an IX81 confocal microscope (Olympus), magnification, 500x, excitation at $543 \mathrm{~nm}$ of (A) Le $\mathrm{C}^{\mathrm{C}}$ as a ligand and (B) 6-O-Su-LeC as a ligand.

incubation with human antibodies. Note that the morphologies shown in Figure 1 are well reproducible from test to test, so the specific shapes of the spots are related to the chemical nature of the glycans, and the abnormalities are not related to the interaction of glycans with antibodies. Staining with a lectin cocktail (instead of antibodies) reproduced the same pattern seen using antibody detection (data not shown). Therefore, we conclude that spot morphology is formed at the stage of glycan immobilization.

In order to significantly decrease or abolish the effect of the glycan on the morphology of the spots (Figure 3), it was next necessary to explore the cause. One possible cause for deviation in spot shape is nonuniform distribution of the substances within the surface layer of the hydrogel. Although immobilization occurs on the surface, the 3D process must be considered. During immobilization, the substance is not only distributed over the surface, but also penetrates into the hydrogel layer covering the glass slide. Indeed, the results of confocal microscopy showed a fluorescent layer several micrometers in thickness (Figure 3), but the thickness proved to be the same for all spots, ideal and with a halo. Moreover, the thickness of the layer in the nucleus zone and in the halo zone was the same (Figure 3B). Therefore, we concluded that the 3D distribution of the solution throughout

Table 1. Variants of buffer composition used for spot morphology improvement.

\begin{tabular}{|l|l|}
\hline $0.005 \%$ Tween-20 in SPB & $18 \%$ sucrose $+0.005 \%$ Tween-20 (in SPB) \\
\hline $0.005 \%$ CHAPS in SPB & $9 \%$ sucrose $+0.005 \%$ Tween-20 (in SPB) \\
\hline $0.005 \%$ Triton X100 in SPB & $18 \%$ fructose $+0.005 \%$ Tween-20 (in SPB) \\
\hline $0.005 \%$ NP40 in SPB & $18 \%$ sucrose $+0.015 \%$ Tween-20 (in SPB) \\
\hline $1 \mathrm{M} \mathrm{NaCl}$ in SPB & $18 \%$ sucrose $+0.015 \%$ NP40 (in SPB) \\
\hline $1.5 \%$ hexadecanol in SPB & $18 \%$ sucrose $+0.01 \%$ CHAPS (in SPB) \\
\hline $0.005 \%$ Tween-20 and $18 \%$ sucrose in SPB & $18 \%$ sucrose $+0.005 \%$ Tween- $20+0.005 \%$ CHAPS (in SPB) \\
\hline PBS, pH7.4 & $18 \%$ sucrose $+0.005 \%$ Tween- $20+0.15 \%$ poly(ethylene oxide) $400 \mathrm{kDa}$ (in SPB) \\
\hline $100 \mathrm{mM}$ borate buffer, pH 8.5 & $18 \%$ sucrose $+0.005 \%$ Tween- $20+0.005 \%$ poly(ethylene oxide) $+1.5 \%$ hexadecanol (in SPB) \\
\hline $18 \%$ sucrose in SPB & $2.5 \%$ sucrose +0.005 Tween-20 (in SPB) \\
\hline $1.5 \mathrm{M}$ betaine in SPB & $10 \%$ inosytol $+0.005 \%$ Tween-20 (in SPB) \\
\hline $4 \% 1$-butyl-3-methylimidazolium tetrafluoroborate in SPB & $5 \%$ inosytol + Tween-20 (in SPB) \\
\hline $0.15 \%$ poly(ethylene oxide) $400 \mathrm{kDa}$ in SPB & $0.005 \%$ Tween- $20+0.005 \%$ CHAPS (in SPB) \\
\hline $0.3 \%$ polyvinylpyrrolidone $400 \mathrm{kDa}$ in SPB & $25 \%$ DMSO $+1 \%$ triethylamine \\
\hline $1 \%$ dextran $40 \mathrm{kDa}$ in SPB & $50 \%$ DMSO $+1 \%$ triethylamine \\
\hline $0.02 \%$ agarose $+0.005 \%$ Tween-20 (in $15 \mathrm{mM} \mathrm{SPB)}$ & $75 \%$ DMSO $+1 \%$ triethylamine \\
\hline $1 \%$ BSA $+0.005 \%$ Tween-20 (in SPB) & $100 \%$ DMSO $+1 \%$ triethylamine \\
\hline
\end{tabular}




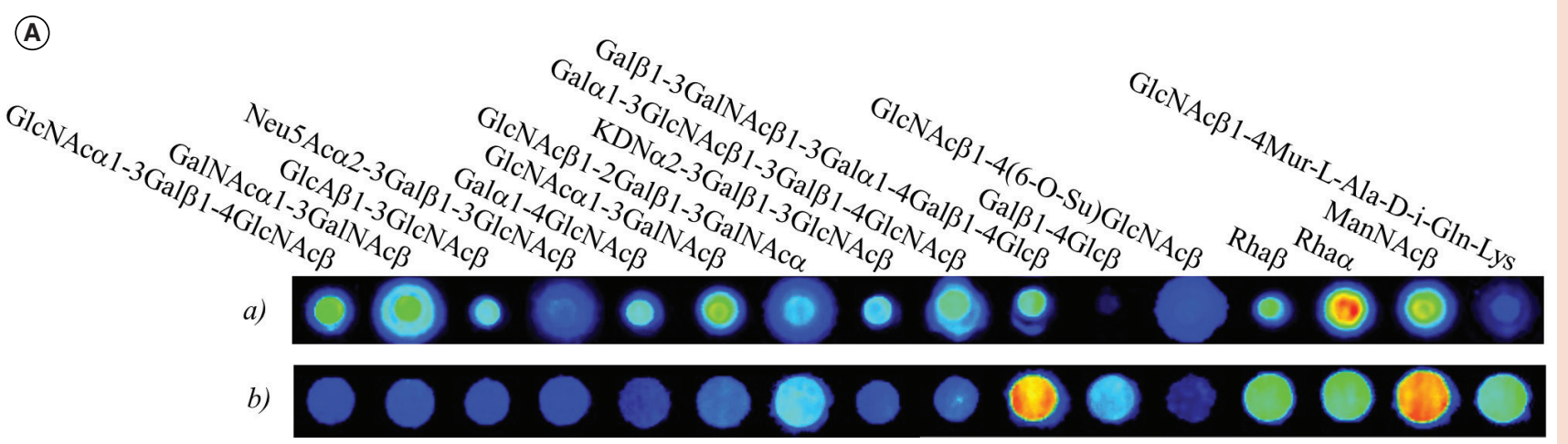

(B)

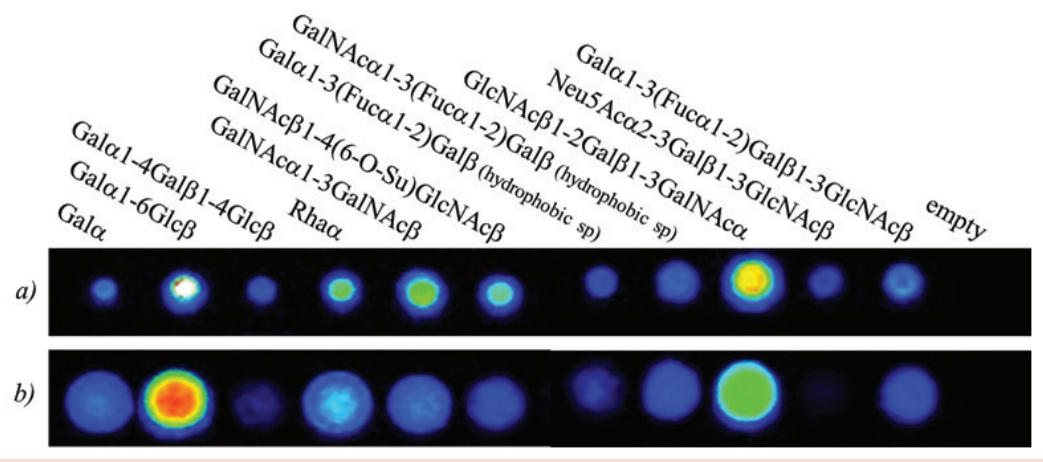

Figure 4. Typical spot morphologies in experiments using a mask or conjugation with polymers. (A) Experiments with a mineral oil mask: (a) regular printing; (b) non-contact printing from a mixture of DMSO:triacetine on a slide coated with vaseline oil:dodecane (2:1). (B) Experiments with polymer-conjugated glycans: (a) non-conjugated glycans as aminospaced glycosides; (b) the same set of PAA-glycoconjugates; contact printing. The slides were developed with antibodies.

the hydrogel was not the cause of the variation.

Another potential cause for variation of spot morphology was unequal spreading of the drop on the slide surface followed by uneven evaporation of the solvent. This process was studied immediately after application of solution drops on the surface by a contact pin using light microscopy. Indeed, the drops dried unevenly and at different rates. The glycan was at a relatively low concentration compared to the buffer salts, but surprisingly, the glycan significantly affected the solution properties. The concentration of the glyco component dramatically increases during drying, resulting in crystallization of the buffer salts.

We supposed that preventing rapid drying would ensure formation of identical spots because the ligand would have more time for covalent immobilization before the physical factors related to drying begin. One way to ensure this was to increase the relative humidity during printing. However, a relative humidity $\geq 50 \%$ increased the rate of hydrolysis of NHS groups on the slide surface and caused intense condensation inside the printing area. Therefore, we had to look for other approaches.

\section{Changes in buffer composition}

A number of studies on drop drying during preparation of DNA and protein microarrays have been performed [4-9]; in particular, the changes in the composition of the printing buffer have been analyzed [4, 9]. We tested several additives (Table 1) for either leveling differences between the drying processes or considerably decreasing the rate of drying. These additives included (a) detergents that do not slow the evaporation, but are expected to ensure unified drop size through surface tension leveling; (b) polyhydroxylated compounds that are very similar to glycans and are capable of forming slowly drying cells; and (c) betain and polymers that are capable of retaining water and, hence, might ensure even evaporation. In fact, the addition of detergents (Tween-20, CHAPS, Triton X100, NP40, and hexadecanol-1), polyxydroxylated compounds (sucrose, fructose, and inositol), zwitter ions (betain), polymers (polyethyleneoxide, polyvinylpyrrolidone, polyacrylamide, dextran, and BSA), or their combinations, as well as DMSO, at different $\mathrm{pH}$ values did not lead to the desired unification of the spots (results not shown).

\section{Printing through a mask}

Another approach to prevent drop drying is pre-coating the slide with a thin layer of low-density hydrophobic liquid, followed by non-contact printing of glycan solutions. The regular solvent for glycans is phosphate buffer, but DMSO is also a good solvent for most glycans. We chose to use DMSO-triacetin because the density of the regular print buffer is very low and is dispersed into the mask (the mask thickness is about $16 \mu \mathrm{m})$. A drop of solution pressed out of the capillary breaks through the mask and deposits on the slide surface. All of the drops deposited under the oil mask had equal volumes and occupied equal areas. The drop size was constant, regardless of the printing time, due to 
the absence of evaporation. As a result, round, even spots were formed. Figure 4 shows that the spots obtained using this technique had even signal distribution, equal sizes, and the absence of a halo or donut effect (a similar technique for preparation of polymer microarrays has been described [10]). An additional advantage of printing through a mask is the protection of NHS slides from direct contact with moisture in the air, resulting in enhanced signals. Finally, the use of a mask does not affect the background signal, and the oil is easy to remove with detergents.

\section{Printing of glycans in a poly-} mer-conjugated form

The attachment to a polymer chain levels out the physicochemical properties of glycans, some of which have significant differences in charge, hydrophobicity, and size in the native state (compare 3',4'-di-O-Su-Lac, Fuc, and biantennary undecasaccharide). Poly-N-2-hydroxyethylacrylamide (PAA), a derivative of $30-\mathrm{kDa}$ polyacrylamide [11,12], was selected as the polymer carrier. Importantly, the primary hydroxyl groups of the polymer are capable of covalently binding to the NHS-activated slide due to their sufficient nucleophilicity. Glycopolymers have a significantly improved spot morphology compared to monovalently attached glycans (Figure 4).

Additional advantages of immobilization of PAA-bound glycans include the following: (1) the reactivities of glycopolymers are equalized due to the $\mathrm{CH}_{2} \mathrm{CH}_{2} \mathrm{OH}$ pendant groups, so the degree of immobilization is independent of the glycan, whereas immobilization of monomeric glycans inevitably gives rise to different degrees of attachment of different glycans to the surface; (2) it is easy to introduce fluorescein labels for quantitation (less than 1 mol\% in the composition of the glycopolymer); as a result, immobilization of each glycan in each spot can be checked [US2013288928A1, 2013]; moreover, quantitative determination of the immobilized glycans appears to be possible because the label-to-glycan ratio in the polymer is strictly controlled.

In summary, printing polymer-conjugated glycans or printing through a mineral oil mask yields dramatically improved arrays, where all the spots are practically identical in size and morphology. The choice of the first or the second method for improving spot morphology is case-dependent. Printing through the mask is more technically complicated, while printing with PAA-derivatives requires synthesis of the corresponding library.

\section{Author Contributions}

All authors performed the experiments, interpreted the data, wrote the paper, and gave final approval to manuscript.

\section{Funding Sources}

This study was supported by the European Commission Marie Curie Program for support of the EuroGlycoArrays ITN project (Maksim Navakouski and Kowa Chen), EU-FP7 GlycoBioM (Ola Blixt), and the Russian Science Foundation (project No. 14-5000131) (Nadezhda Shilova, Nailya Khasbiullina, Nicolai Bovin).

\section{Acknowledgments}

This study was supported by the European Commission Marie Curie Program for support of the EuroGlycoArrays ITN project (Maksim Navakouski and Kowa Chen), EU-FP7 GlycoBioM (Ola Blixt), and the Russian Science Foundation (project No. 14-5000131) (Nadezhda Shilova, Nailya Khasbiullina, Nicolai Bovin).

\section{References}

1. Rillahan, C.D. and Paulson, J.C. Glycan microarrays for decoding the glycome. 2011 Annu. Rev. Biochem. 80:797-823.

2. Jacob, F., Goldstein, D.R., Huflejt, M., Bovin, N., Pochechueva, T., Spengler, M., Caduff, R., Fink, D. and Heinzelmann-Schwarz, V. 2012 Serum anti-glycan antibody detection of epithelial ovarian cancers by using a printed glycan array. Int. J. Cancer. 130:138-146.

3. Blixt, O., Head, S., Mondala, T., Scanlan, C., Huflejt, M.E., Alvarez, R., Bryan, M.C., Fazio, F., Calarese, D., Stevens, J., Razi, N., Stevens, D.J., Skehel, J.J., van Die, I., Burton, D.R., Wilson, I.A., Cummings, R., Bovin, N., Wong, C.H. and Paulson, J.C. 2004 Printed covalent glycan array for ligand profiling of diverse glycan binding proteins. Proc. Natl. Acad. Sci. USA 101:17033-17038.

4. Dugas, V., Broutin, J. and Souteyrand, E. 2005 Droplet evaporation study applied to DNA chip manufacturing. Langmuir 21:9130-9136.

5. Liu, G., Zhang, C., Zhao, J. and Zhu, Y. 2008 Study of the morphology of the three-phase contact line and its evolution by morphological examination after droplet evaporation of aqueous polymer solutions. Langmuir 24:7923-7930.

6. McHale, G., Aqil, S., Shirtcliffe, N.J., Newton, M.I. and Erbil, H.Y. 2005 Analysis of droplet evaporation on a superhydrophobic surface. Langmuir 21:11053-11060.

7. Fang, Y., Frutos, A.G. and Lahiri, J. 2002 Membrane protein microarrays. J. Am. Chem. Soc. 124:2394-2395.

8. Scurr, D.J., Horlacher, T., Oberli, M.A., Werz, D.B., Kroeck, L., Bufali, S., Seeberger, P.H., Shard, A.G. and Alexander, M.R. 2010 Surface characterization of carbohydrate microarrays. Langmuir 26:17143-17155.

9. Deng, Y., Zhu, X.Y., Kienlen, T. and Guo, A. 2006 Transport at the air/water interface is the reason for rings in protein microarrays. J. Am. Chem. Soc. 128:2768-2769.

10. Liberski, A., Zhang, R. and Bradley, M. 2009 Inkjet fabrication of polymer microarrays and grids - solving the evaporation problem. Chem. Commun. 334-336.

11. Bovin, N.V. 2002 Neoglycoconjugates: trade and art, p. 143-160. In: K.Drickamer and A.Dell (Eds.), Glycogenomics: the Impact of Genomics and Informatics on Glycobiology. Biochem. Soc. Symp. 69. Portland Press, Cambridge.

12. Bovin, N.V. 1998 Polyacrylamide-based glycoconjugates as tools in glycobiology. Glycoconj. J. 15:431-446.

First draft submitted: 25 July 2017; Accepted for publication: 15 February 2018.

Address correspondence to Nicolai Bovin, Shemyakin-Ovchinnikov Institute of Bioorganic Chemistry, Russian Academy of Sciences, MiklukhoMaklaya 16/10, 117997 Moscow, Russia. E-mail: professorbovin@yandex.ru

To purchase reprints of this article, contact: s.cavana@future-science.com 